\title{
Performances de ponte et caractéristiques des œufs de la pintade locale (Numida meleagris) en système de conduite améliorée dans la région Centre du Burkina Faso
}

\author{
R. Sanfo ${ }^{1 *}$ H. Boly ${ }^{1}$ L. Sawadogo ${ }^{2}$ O. Brian ${ }^{3}$
}

\begin{abstract}
Mots-clés
Volaille - Numida meleagris Caractéristiques de l'œuf - Production d'œufs - Performance de ponte Burkina Faso.
\end{abstract}

\begin{abstract}
Résumé
Les performances de ponte et les caractéristiques des œufs de 200 pintades locales (Numida meleagris) couvertes par 100 mâles ont été étudiées au Burkina Faso. L'âge moyen à l'entrée en ponte a été de 5,7 mois avec un poids moyen de 931,9 $\pm 85,3$ g. Les deux cycles de ponte étaient situés en saisons sèches, avec des durées de 4,8 mois pour le premier et de 7,5 mois pour le second, avec respectivement les taux de ponte de 21,6 et de 58,7 p. 100, et les performances moyennes de 31,1 et 121,5 œufs par pintade. Le poids moyen des œufs a été de 29,2 $\pm 1,7 \mathrm{~g}$ au premier cycle contre 37,8 $\pm 3,5 \mathrm{~g}$ au second. Les dimensions moyennes des œufs ont été de 47,1 $\pm 1,9 \mathrm{~mm}$ pour la longueur et de $36,5 \pm 1,3 \mathrm{~mm}$ pour le grand diamètre. L'indice moyen de forme a été plus élevé chez les œufs du second cycle que chez ceux du premier $(0,79 \pm 0,05$ contre $0,76 \pm 0,02)$. L'équation de régression liant le poids de I'œuf $(\mathrm{Y})$ à sa longueur $(\mathrm{L})$ et à son grand diamètre $(\mathrm{Gd})$ a été de : $Y(g)=-74,22+0,91 \mathrm{~L}(\mathrm{~mm})+1,83 \mathrm{Gd}(\mathrm{mm})\left(\mathrm{R}^{2}=0,96\right.$; erreur type =1,47). Les nuances de coloration des coquilles (rousse $=15,7$ p. 100 ; blanche $=$ 19,7 p. 100 ; teintée $=64,6$ p. 100) ont été répertoriées pour l'ensemble des œufs. Le taux de mortalité intracoquille a été de 25,7 p. 100 avec 17,5 p. 100 pour les embryons et 8,2 p. 100 pour les pintadeaux. Des taux de fertilité de 82,7 p. 100 et d'éclosion de 74,3 p. 100 ont été enregistrés.
\end{abstract}

\section{INTRODUCTION}

L'élevage de la pintade locale (Numida meleagris) est une pratique courante au Burkina Faso (13). L'effectif du cheptel, estimé à plus de 6,5 millions d'animaux, est assez révélateur de son importance et indique que la pintade est loin d'être marginale parmi les espèces avicoles. Elle occupe le second rang au plan numérique après le poulet et intervient dans la nutrition, les rapports sociaux et surtout dans la constitution des revenus ménagers, principalement en milieu rural.

En dépit de cette importance, la pintade reste un sujet de seconde zone qui se manifeste par le peu d'investissements zoo-

1. Institut national de l'environnement et des recherches agricoles / Kamboinsé, département Productions animales, 01 BP 476, Ouagadougou 01, Burkina Faso. 2. Université d'Ouagadougou, Burkina Faso.

3. Swedish University of Agricultural Sciences, Department of Animal Nutrition and Management, Uppsala, Sweden.

* Auteur pour la correspondance

Tél. : +22650319229

E-mail : r_sanfo@yahoo.fr vétérinaires, tant des pouvoirs publics que des éleveurs : elle doit son alimentation essentiellement à sa capacité à glaner, son abreuvement est un problème récurrent et son statut sanitaire n'est guère meilleur, surtout au stade de pintadeau, car basé essentiellement sur la pharmacopée traditionnelle. Dans le domaine de l'habitat, les abris de fortune ne lui permettent ni une hygiène convenable, ni une protection contre les prédateurs et les intempéries. En dépit du caractère frileux des pintadeaux (8), le système de chauffage de leur habitat reste inexistant. Ce système d'élevage, qui tient plus de la cueillette que de la production proprement dite, serait corollaire de la faible productivité, y compris en œufs, de la pintade locale dont l'exploitation à grande échelle est fortement limitée par le caractère essentiellement saisonnier de la ponte.

Le présent travail vise à étudier les performances de ponte de la pintade locale (taux de ponte et son évolution), les caractéristiques des œufs (poids, dimensions) et leurs relations avec les pintadeaux (taux de fertilité, mortalité embryonnaire et taux d'éclosion), la mortalité des pintadeaux et leur croissance dans un environnement contrôlé, à la fois sur les aspects de l'habitat, de l'abreuvement de l'alimentation et de la santé. 


\section{MATERIEL ET METHODES}

L'étude a été conduite dans la zone nord soudanienne du Burkina Faso, au Centre de recherches et de formation agricole (CRFA) de Gampéla. Le Centre est situé à une vingtaine de kilomètres à l'est d'Ouagadougou sur l'axe Ouagadougou Fada N'Gourma.

Le lot de pintades considérées dans cette étude était issu d'une incubation artificielle, conduite au mois d'août, de 2500 œufs récoltés dans la région Centre. Les pintadeaux éclos ont été identifiés par des boucles alaires et suivis pour l'évolution pondérale et la mortalité. Ils ont été gardés de 0 à 8 semaines dans des éleveuses Attesta de fabrication locale, maintenues à une température moyenne de $37^{\circ} \mathrm{C}$ à l'aide de lampes à pétrole. Après huit semaines, ils ont été transférés dans des habitats pour adultes. Ils ont été nourris ad libitum avec des aliments de type industriel (tableau I) avec trois types de rations : démarrage (0 à 8 semaines), croissance ( 9 à 20 semaines) et ponte (au-delà de 20 semaines).

A cinq mois d'âge, les pintades ont été sexées par l'observation de la forme et de la consistance des éminences génitales (1) et le lot parental (300 pintades dont 200 femelles et 100 mâles) a été constitué par un tirage aléatoire. Ce lot a été divisé en quatre répétitions, de 50 femelles et 25 mâles chacune, qui ont été suivies pour la ponte. Un vaccin antiNewcastle a été administré aux pintadeaux à trois semaines d'âge, puis repris tous les six mois avec un déparasitage interne à l'aide du trichomonacide ou vermifuge spécial pintade.

Les œufs pondus par le lot parental ont été quotidiennement récoltés, identifiés par des numéros, puis calibrés. Le poids a été déterminé à l'aide d'une balance électronique de $180 \mathrm{~g} \pm 0,1 \mathrm{mg}$ (AND ${ }^{\circledR}$ ) et les dimensions ont été mesurées à l'aide d'un calibreur électronique de $150 \pm 0,01 \mathrm{~mm}\left(\mathrm{CE}^{\circledR}\right)$. L'indice de forme de l'œuf a été calculé par le rapport du grand diamètre sur la longueur (grand diamètre/longueur) de l'œuf (12).

Un lot de 800 œufs, retenus par un tirage aléatoire et préalablement conservés dans un réfrigérateur sur une durée maximale de 10 jours à une température de $15^{\circ} \mathrm{C}$ et à une humidité relative de $60 \mathrm{p} .100$, a été mis à incuber. L'incubation a été conduite à une température moyenne de $38,2^{\circ} \mathrm{C}$ et à une humidité relative de 60 p. 100 contre $37,8^{\circ} \mathrm{C}$ et 80 p. 100 d'humidité relative pour l'éclosion (6). Le transfert des œufs de l'incubateur à l'éclosoir a été effectué au 24 e jour d'incubation. Un incubateur et un éclosoir de type 2-500 FH ont été utilisés à cet effet. Ces appareils étaient munis de systèmes de ventilation forcée et l'incubateur comportait en outre un système de retournement automatique.

Deux mirages ont été effectués à l'aide d'une mireuse électrique de $40 \mathrm{~W}$ aux $9^{\mathrm{e}}$ et $24^{\mathrm{e}}$ jours (6). Ils ont permis de déterminer respectivement les taux de fertilité et de mortalité embryonnaire précoce. Les mortalités à l'éclosion ont été observées au 7 jour de la mise des œufs dans l'éclosoir.

Source : Centre national de développement de l'aviculture

* En pourcentage de la MS
Les variables de l'incubation ont été calculées selon les formules appliquées par Ayorinde et Ayeni (6) avec :

taux de fertilité $(\%)=$ (nb. d'œufs fertiles / nb. total d'œufs mis à incuber) $\mathrm{x} 100 \mathrm{et}$

taux réel d'éclosion $(\%)=(\mathrm{nb}$. de pintadeaux éclos / nb. d'œufs fertiles) x 100 .

L'analyse bromatologique des aliments a été faite par le Centre national de développement de l'aviculture (tableau I). Les quantités consommées $\left(\mathrm{Q}_{\mathrm{C}}\right)$ ont été évaluées à l'aide de pesées quotidiennes des aliments, en faisant la différence entre les quantités distribuées $\left(\mathrm{Q}_{\mathrm{D}}\right)$ et les quantités refusées $\left(Q_{R}\right)$. Les indices de consommation ont été calculés avec la formule :

$\mathrm{Q}_{\mathrm{C}} / \mathrm{G}_{\mathrm{P}}$ ou encore $\left(\mathrm{Q}_{\mathrm{D}}-\mathrm{Q}_{\mathrm{R}}\right) / \mathrm{G}_{\mathrm{P}}$

où $\mathrm{G}_{\mathrm{P}}$ est le gain de poids durant la période de suivi.

Le suivi de l'évolution pondérale des pintadeaux a été réalisé avec une balance électronique $(5 \mathrm{~kg} \pm 5 \mathrm{~g})\left(\mathrm{EKS}^{\circledR}\right)$. Les taux mensuels de ponte ont été calculés selon la formule :

\section{$[\mathrm{N} /(\mathrm{PK})] \mathrm{x} 100$}

où $\mathrm{N}$ est le nombre total d'œufs produits, $\mathrm{P}$ le nombre de pondeuses et $\mathrm{K}$ le nombre de jours de ponte (20).

Les données recueillies ont été analysées à l'aide du logiciel StatView, version 4.5. Les moyennes ont été comparées à l'aide du test $t$ de Student au seuil de signification de 5 p. 100. Le niveau de signification des coefficients de corrélation a été déterminé par le test $Z$ au seuil de 5 p. 100.

\section{RESULTATS}

\section{Performances de ponte}

Les données sur la consommation alimentaire (quantités d'aliments consommés et indices de consommation) sont présentées dans le tableau II.

Aucune mortalité n'a été enregistrée dans le lot de pintades destinées à la ponte au cours de l'essai. L'âge moyen à la première entrée en ponte a été de 22,8 semaines avec un poids vif moyen de 931,9 \pm 85,3 g. La deuxième entrée en ponte a été enregistrée à 16,2 mois d'âge. Le tableau III montre l'évolution bihebdomadaire du poids du cheptel.

Le premier cycle de ponte s'est installé en saison sèche, du 13 janvier au 25 mars (2004), pour une durée totale de 4,8 mois. Le second cycle a de même démarré en saison sèche (le 15 décembre 2004) et s'est poursuivi jusqu'en début d'hivernage (le 27 juillet 2005), avec une durée totale de 7,5 mois. L'intervalle entre les deux cycles de ponte a été de 8,7 mois.

\section{Tableau I}

Valeurs bromatologiques mesurées par le Laboratoire national d'élevage

\begin{tabular}{|c|c|c|c|c|c|c|c|c|c|c|}
\hline Type d'aliment & $\begin{array}{l}\text { MS } \\
(\%)\end{array}$ & $\begin{array}{c}\text { MAT }^{*} \\
(\%)\end{array}$ & $\begin{array}{c}\mathrm{MG}^{*} \\
(\%)\end{array}$ & $\begin{array}{l}C^{*}{ }^{*} \\
(\%)\end{array}$ & $\begin{array}{c}\text { EM } \\
\text { (Kcal) }\end{array}$ & $\begin{array}{l}\mathrm{Ca}^{*} \\
(\%)\end{array}$ & $\begin{array}{l}P^{*} \\
(\%)\end{array}$ & $\begin{array}{l}\text { Lys * } \\
\text { (\%) }\end{array}$ & $\begin{array}{c}\text { Met }^{*} \\
(\%)\end{array}$ & $\begin{array}{c}\text { Cys * } \\
(\%)\end{array}$ \\
\hline Démarrage & 89,5 & 19,8 & 4,4 & 4,0 & 2970 & 1,1 & 0,7 & 1,1 & 0,5 & 0,3 \\
\hline Croissance & 89,7 & 16,5 & 4,1 & 5,3 & 2900 & 1,4 & 0,6 & 0,7 & 0,3 & 0,4 \\
\hline Ponte & 90,3 & 17,0 & 3,8 & 3,8 & 2750 & 4,0 & 0,6 & 0,8 & 0,4 & 0,4 \\
\hline
\end{tabular}

MS : matière sèche ; MAT : matières azotées totales ; MG : matières grasses ; CB : cellulose brute ; EM : énergie métabolisable 
L'évolution des taux de ponte selon le cycle est présentée dans les tableaux IV et V. Le taux moyen de ponte a été de 21,6 p. $100 \mathrm{au}$ premier cycle et de 58,7 p. 100 au second (moyenne $=40,2$ p. 100). Le taux le plus bas (15,3 p. 100) a été noté à la fin du premier cycle au mois de mai. Le taux de ponte a culminé à 82,8 p. 100 au mois

\section{Tableau II}

Consommation alimentaire selon les périodes

\begin{tabular}{lcccc} 
Classe d'âge (semaines) & $\mathbf{0 - 6}$ & $\mathbf{7 - 1 2}$ & $\mathbf{1 3 - 1 8}$ & $\mathbf{0 - 2 4}$ \\
\hline Aliments consommés (g/j) & 14,0 & 44,5 & 75,6 & 44,7 \\
Indice de consommation & 3,5 & 4,0 & 4,7 & 4,0
\end{tabular}

d'avril, ce qui correspond au $10^{\mathrm{e}}$ mois de l'entrée en ponte et au $5^{\mathrm{e}}$ mois du second cycle. Les valeurs de 31,1 et de 121,5 œufs par pintade ont été enregistrées respectivement au premier et au second cycle de ponte. L'augmentation du nombre d'œufs par pintade du premier au second cycle a été de 290,7 p. 100.

\section{Caractéristiques des æuufs}

Le premier œuf pondu avait un poids de 21,0 g, une longueur de 40,2 $\mathrm{mm}$ et un grand diamètre de $30,6 \mathrm{~mm}$. Les données métriques des œufs (poids, longueur, diamètre, indice de forme) sont détaillées dans le tableau IV pour le premier cycle et dans le tableau $\mathrm{V}$ pour le second.

Le poids moyen (Y) de l'œuf est lié à sa longueur (L) et à son grand diamètre $(\mathrm{Gd})$ par les équations de régression :

\section{Tableau III}

Evolution du poids du cheptel selon la période de pesée

\begin{tabular}{|c|c|c|c|c|c|c|c|c|c|c|c|c|}
\hline $\begin{array}{l}\text { Date de pesée } \\
\text { (semaines) }\end{array}$ & 0-2 & $3-4$ & 5-6 & $7-8$ & 9-10 & $11-12$ & $13-14$ & $15-16$ & $17-18$ & 19-20 & $21-22$ & $22-23$ \\
\hline $\begin{array}{l}\text { Poids des } \\
\text { femelles (g) }\end{array}$ & $\begin{array}{l}25,8^{a} \\
\pm 3,1\end{array}$ & $\begin{array}{r}50,1^{b} \\
\pm 15,7\end{array}$ & $\begin{array}{r}88,1^{c} \\
\pm 35,5\end{array}$ & $\begin{array}{l}124,9^{d} \\
\pm 52,9\end{array}$ & $\begin{array}{l}208,1^{\mathrm{e}} \\
\pm 84,4\end{array}$ & $\begin{array}{l}292,1^{f} \\
\pm 96,5\end{array}$ & $\begin{array}{c}414,09 \\
\pm 114,4\end{array}$ & $\begin{array}{l}541,1^{\mathrm{h}} \\
\pm 153,1\end{array}$ & $\begin{array}{c}673,8^{i} \\
\pm 167,2\end{array}$ & $\begin{array}{c}781,3^{j} \\
\pm 172,3\end{array}$ & $\begin{array}{c}869,6^{k} \\
\pm 151,2\end{array}$ & $\begin{array}{c}931,9 \\
\pm 143,8\end{array}$ \\
\hline $\begin{array}{l}\text { Poids des } \\
\text { mâles (g) }\end{array}$ & $\begin{array}{l}25,5^{\mathrm{a}} \\
\pm 2,4\end{array}$ & $\begin{array}{l}45,7^{b} \\
\pm 13,8\end{array}$ & $\begin{array}{l}77,2^{\mathrm{c}} \\
\pm 29,3\end{array}$ & $\begin{array}{l}121,0^{d} \\
\pm 52,3\end{array}$ & $\begin{array}{l}190,2^{f} \\
\pm 76,7\end{array}$ & $\begin{array}{l}265,6^{\mathrm{g}} \\
\pm 88,9\end{array}$ & $\begin{array}{l}352,2^{\mathrm{h}} \\
\pm 114,9\end{array}$ & $\begin{array}{c}442,3^{i} \\
\pm 171,8\end{array}$ & $\begin{array}{c}603,2^{j} \\
\pm 171,1\end{array}$ & $\begin{array}{c}707,8^{k} \\
\pm 189,9\end{array}$ & $\begin{array}{l}762,2^{1} \\
\pm 210,0\end{array}$ & $\begin{array}{l}853,2^{\mathrm{m}} \\
\pm 183,7\end{array}$ \\
\hline
\end{tabular}

Les nombres portant en exposant les mêmes lettres dans la même colonne ne sont pas significativement différents au seuil de $5 \%$.

\section{Tableau IV}

Taux de ponte et données métriques des œufs au premier cycle

\begin{tabular}{|c|c|c|c|c|c|c|}
\hline & $\begin{array}{c}\text { Janvier } \\
(n=200)\end{array}$ & $\begin{array}{l}\text { Février } \\
(n=200)\end{array}$ & $\begin{array}{c}\text { Mars } \\
(n=200)\end{array}$ & $\begin{array}{c}\text { Avril } \\
(n=200)\end{array}$ & $\begin{array}{c}\text { Mai } \\
(n=200)\end{array}$ & Moyenne \\
\hline Taux de ponte (\%) & 25,1 & 26,6 & 24,0 & 17,2 & 15,3 & 21,6 \\
\hline Poids des œufs (g) & $26,9^{a} \pm 2,0$ & $29,1^{b} \pm 1,9$ & $30,4^{b} \pm 1,5$ & $30,0^{b} \pm 1,3$ & $29,7^{b} \pm 1,7$ & $29,2 \pm 1,7$ \\
\hline Longueur (mm) & $44,2^{\mathrm{a}} \pm 2,0$ & $45,0^{\mathrm{a}} \pm 1,9$ & $45,4^{\mathrm{a}} \pm 1,3$ & $45,8^{a} \pm 0,9$ & $45,7^{\mathrm{a}} \pm 0,7$ & $45,9 \pm 1,4$ \\
\hline Diamètre (mm) & $33,6^{\mathrm{a}} \pm 1,2$ & $34,4^{\mathrm{a}} \pm 1,4$ & $34,5^{\mathrm{a}} \pm 0,9$ & $35,1^{\mathrm{a}} \pm 0,7$ & $35,2^{\mathrm{a}} \pm 0,8$ & $34,9 \pm 1,0$ \\
\hline Indice de forme & $0,76^{a} \pm 0,02$ & $0,76^{a} \pm 0,03$ & $0,76^{a} \pm 0,02$ & $0,77^{\mathrm{a}} \pm 0,01$ & $0,77^{a} \pm 0,02$ & $0,76^{a} \pm 0,02$ \\
\hline
\end{tabular}

$\mathrm{n}$ : nombre de femelles

Les nombres portant en exposant les mêmes lettres sur la même ligne ne sont pas significativement différents au seuil de $5 \%$.

Tableau V

Taux de ponte et données métriques des œufs au second cycle

\begin{tabular}{|c|c|c|c|c|c|c|c|c|c|}
\hline & $\begin{array}{c}\text { Décembre } \\
(n=200)\end{array}$ & $\begin{array}{l}\text { Janvier } \\
(\mathrm{n}=200)\end{array}$ & $\begin{array}{l}\text { Février } \\
(n=200)\end{array}$ & $\begin{array}{c}\text { Mars } \\
(n=200)\end{array}$ & $\begin{array}{c}\text { Avril } \\
(n=200)\end{array}$ & $\begin{array}{c}\text { Mai } \\
(n=200)\end{array}$ & $\begin{array}{c}\text { Juin } \\
(n=200)\end{array}$ & $\begin{array}{c}\text { Juillet } \\
(n=200)\end{array}$ & Moyenne \\
\hline Taux de ponte (\%) & 34,1 & 57,9 & 57,5 & 66,7 & 82,8 & 74,7 & 63,5 & 32,7 & 58,7 \\
\hline Poids des œufs (g) & $38,6^{a} \pm 2,9$ & $38,1^{\mathrm{a}} \pm 3,2$ & $38,2^{a} \pm 3,6$ & $37,4^{\mathrm{a}} \pm 3,3$ & $37,5^{\mathrm{a}} \pm 3,8$ & $36,9^{a} \pm 3,7$ & $36,8^{a} \pm 3,7$ & $36,7^{a} \pm 3,7$ & $37,8 \pm 3,5$ \\
\hline Longueur (mm) & $48,9^{a} \pm 2,1$ & $47,8^{a} \pm 2,3$ & $48,6^{a} \pm 1,9$ & $48,8^{a} \pm 2,6$ & $47,5^{\mathrm{a}} \pm 2,4$ & $47,4^{a} \pm 2,5$ & $48,3^{a} \pm 2,5$ & $48,4^{\mathrm{a}} \pm 2,7$ & $48,2 \pm 2,4$ \\
\hline Diamètre (mm) & $37,3^{a} \pm 1,0$ & $37,4^{\mathrm{a}} \pm 1,1$ & $37,7^{\mathrm{a}} \pm 1,5$ & $37,2^{\mathrm{a}} \pm 1,4$ & $38,7^{\mathrm{a}} \pm 2,7$ & $37,0^{\mathrm{a}} \pm 1,5$ & $37,7^{\mathrm{a}} \pm 1,7$ & $38,2^{a} \pm 1,8$ & $38,1 \pm 1,6$ \\
\hline Indice de forme & $0,77^{a} \pm 0,03$ & $0,78^{a} \pm 0,04$ & $0,79^{a} \pm 0,06$ & $0,79^{a} \pm 0,06$ & $0,82^{\mathrm{a}} \pm 0,07$ & $0,78^{a} \pm 0,04$ & $0,78^{a} \pm 0,04$ & $0,79^{a} \pm 0,03$ & $0,79 \pm 0,05$ \\
\hline
\end{tabular}


- simples : $\mathrm{Y}(\mathrm{g})=-64,41+2,74 \mathrm{Gd}(\mathrm{mm})\left[\mathrm{R}^{2}=0,91 ;\right.$ erreur type $=2,24]$;

$\mathrm{Y}(\mathrm{g})=-50,77+1,82 \mathrm{~L}(\mathrm{~mm})\left[\mathrm{R}^{2}=0,86 ;\right.$ erreur type $\left.=2,81\right]$;

- multiple : $\mathrm{Y}(\mathrm{g})=-74,22+0,91 \mathrm{~L}(\mathrm{~mm})+1,83 \mathrm{Gd}(\mathrm{mm})\left[\mathrm{R}^{2}=0,96\right.$; erreur type $=1,47)$.

Il ressort des équations de régression simples que le grand diamètre détermine mieux le poids de l'œuf que la longueur $\left(\mathrm{R}^{2}=0,91\right.$ contre $0,86)$. Le poids de l'œuf a augmenté avec le rang du mois de ponte. Ces deux variables ont été positivement et significativement $(\mathrm{p}<0,05)$ corrélées $(r=0,71)$ au seuil de 5 p. 100.

Trois nuances de coloration (rousse, blanche, teintée) ont été répertoriées, avec 15,7 p. 100 de coloration rousse, 19,7 p. 100 de blanche et 64,6 p. 100 de teintée. Il n'y a pas eu de différence significative ( $p>0,05)$ entre le poids des œufs selon la souche.

Un taux de fertilité de 82,7 p. 100 a été relevé au niveau des œufs. Les observations des taux de fertilité n'ont pas été effectuées selon le poids et la couleur des œufs. Les valeurs de 25,7 et de 74,3 p. 100 ont été notées respectivement pour le taux de mortalité embryonnaire et le taux réel d'éclosion. La mortalité précoce (durant l'incubation) a été de 17,5 p. 100 et la mortalité à l'éclosion (durant l'éclosion) de 8,2 p. 100 .

\section{- DISCUSSION}

Le nombre élevé des femelles et la faible sex-ratio de l'essai avaient pour but d'augmenter les chances de disposer d'œufs fertiles en nombre suffisant pour l'incubation. L'amélioration des conditions d'élevage a induit une baisse notable de l'âge à l'entrée en ponte de la pintade locale : celle-ci s'est en effet produite en deçà de l'intervalle de 7 à 10,5 mois relevé en milieu traditionnel au Burkina Faso (11), ce qui correspond à des baisses respectives de 18,6 et de 45,7 p. 100 . Cet âge à l'entrée en ponte a été également plus précoce de 0,7 mois que celui de 6,4 mois observé au Nigeria dans un élevage amélioré (15).

L'âge de l'entrée en ponte de la pintade locale a été le même que celui de la poule locale. L'âge de 6,3 mois a été rapporté pour la pintade en conditions d'alimentation améliorées au Nigeria (3) et de 6,3 $\pm 0,4$ mois pour la poule locale (Gallus gallus) en conditions traditionnelles au Burkina Faso (17).

Le poids vif de la pintade locale à l'entrée en ponte a été comparable à ceux rapportés par Ayorinde et coll. au Nigeria (7) pour toutes les variétés phénotypiques de pintades identifiées : lilas $(962 \pm 175 \mathrm{~g})$, noire $(950 \pm 197 \mathrm{~g})$, gris perlée $(979 \pm 149 \mathrm{~g})$ et blanche $(947$ $\pm 237 \mathrm{~g})$.

La pintade locale est communément reconnue comme une espèce dont la ponte survient en saison pluvieuse dans les zones subhumides à arides (1). Les résultats de cette étude ont montré que cette perception ne pouvait être généralisée comme le prouvait la présence de pintadeaux dans le milieu villageois en saison sèche. Le désaisonnement ainsi observé de la ponte de la pintade locale pourrait s'expliquer par l'amélioration des conditions alimentaires. Ces pontes de contre-saison sont très encourageantes et constituent une solution au développement et à l'exploitation à large échelle de cette espèce eu égard à la grande consommation de ses œufs et de sa viande (4). Ces résultats appellent toutefois des mesures d'accompagnement pour la conservation des œufs qui se détériorent rapidement dans les conditions drastiques des facteurs climatiques en saison sèche dans ces régions.

La durée du cycle de ponte est un facteur important dans la production des œufs. Celle-ci va de 3,3 à 6,4 mois $(9,10)$. La durée de la ponte du premier cycle a confirmé les résultats ci-dessus. Celle-ci a été brève, par rapport au second cycle, tant dans le temps que dans l'intensité de ponte et donc du nombre d'œufs produits. Elle pourrait être dû au fait que les pintades entrent nouvellement en ponte et ne seraient pas tout à fait adaptées à cette fonction malgré leur poids, car elles ont été nourries aux aliments industriels.

Pour le second cycle, la durée de ponte a été plus longue que celles rapportées par ces mêmes auteurs $(9,10)$ et son allongement s'expliquerait par les conditions alimentaires améliorées.

Le nombre moyen d'œufs/pintade/an se situe dans l'intervalle de 36 à 110 (16). Dans des conditions alimentaires améliorées, les performances de ponte de la pintade locale sont maintenues en saison sèche, permettant de considérer cette volaille comme une bonne pondeuse, comparée à la poule locale qui est créditée de 50 œufs (13).

Le poids moyen des œufs au premier cycle de ponte relève de la catégorie des œufs légers (inférieur à $30 \mathrm{~g}$ ) (5). Cette caractéristique implique la nécessité d'effectuer un tri pour écarter de l'incubation les œufs de moins de $30 \mathrm{~g}$, selon les recommandations du même auteur.

Le poids moyen de l'œuf au second cycle de ponte a été relativement supérieur à la valeur de $35,7 \pm 2,8 \mathrm{~g}$ rapportée dans l'ouest du Burkina Faso (13), comparable à celui de $37,3 \mathrm{~g}$ pour le cas du Mali central (14) et compris dans l'intervalle de 35,8 à 44,6 g relevé au Nigeria (4).

L'indice de forme de l'œuf est utilisé pour mesurer la résistance mécanique de la coquille (13). Les indices calculés dans les deux cycles de ponte ont été proches de ceux des œufs standard qui sont compris entre 0,72 et 0,75 (12).

Les trois nuances de la coloration de la coquille observées suggèrent l'existence de trois souches de pintades dans la population étudiée et sont conformes aux résultats antérieurs (12). Des investigations ultérieures pourraient être conduites pour la caractérisation desdites souches dans l'optique de leur gestion rationnelle.

Le taux de fertilité a été légèrement supérieur à celui de 72,5 p. 100 rapporté par Sauveur (19). Le niveau réduit de la sex-ratio pourrait expliquer ce fait. La fertilité de l'œuf ne serait donc pas une contrainte au développement de l'élevage de la pintade locale en saison sèche au Burkina Faso. Il faudrait toutefois veiller au respect de la sex-ratio maximale de deux femelles pour un mâle.

Le taux réel d'éclosion est compris dans l'intervalle de 55 à 100 p. 100 (2). Ce taux a toutefois été inférieur à ceux de 85,2 et 82,9 p. 100 observés respectivement en incubations artificielle et naturelle (20). Ce taux moindre pourrait s'expliquer par le fait que la présente étude a porté sur une variété de pintade de format moyen, le taux d'éclosion étant significativement influencé par la variété de la pintade (2). A cet effet, les taux de 86 à 100 p. 100 pour la variété lourde contre 72,9 p. 100 pour la variété légère ont été rapportés au Togo (10).

\section{- CONCLUSION}

Le caractère hivernal de la ponte chez la pintade locale dans la région Centre du Burkina Faso n'est pas absolu. L'amélioration des conditions alimentaires affecte significativement l'âge à l'entrée en ponte et les performances de ponte, induit le désaisonnement et allonge la durée de ponte. Le poids moyen des œufs augmente avec le rang du cycle de ponte. Eu égard à la variabilité pondérale observée, il est nécessaire que les œufs soient soumis à un tri, surtout ceux du premier cycle de ponte, en vue d'obtenir de bons résultats lors de l'incubation artificielle. Des études sur les différentes variétés et souches de la pintade locale restent à conduire dans l'optique de déterminer leurs caractéristiques spécifiques pour une gestion rationnelle. 


\section{BIBLIOGRAPHIE}

1. ANONYME, 1996. Quelques notions sur l'élevage de la pintade. Ouagadougou, Burkina Faso, ministère délégué chargé des Ressources animales, $26 \mathrm{p}$.

2. ASSOUMANE I., GOURO A.S., 1997. L'élevage des pintades au Niger. In : Sonaiya E.B. éd., Proc. Issues in family poultry research and developpement workshop, Mbour, Senegal, 9-13 Dec., p. 254-259.

3. AYENI J.S.O., 1987. Study of grey-breasted guinea fowl (Numida meleagris galeata pallas) in Nigeria. World Poult. Sci. J., 39: 143-151.

4. AYORINDE K.L., 1991. Yield and chemical composition of Cyperus bulb in Kainji Lake Basin. Nig. J. Nutr. Sci., 12: 26-27.

5. AYORINDE K.L., 2004. The spice of life. The seventy-first inaugural lecture. Ilorin, Nigeria, University of Ilorin, $60 \mathrm{p}$.

6. AYORINDE K.L., AYENI J.S.O., 1987. Performance of guinea fowl breeders fed varying levels of Cyperus bulb. J. Anim. Prod., 14: 139-145.

7. AYORINDE K.L., OLUYEMI J.A., AYENI J.S.O., 1988. Growth performance of four indigenous helmeted guinea fowl varieties (Numida meleagris galeata pallas) in Nigeria. Bull. Anim. Health Prod. Afr., 36: 356-360.

8. CASTAING J., 1979. Aviculture et petits élevages. Paris, France, J.-B. Baillière, 313 p.

9. CHRYSOSTOME C., 1992. The use of termites for raising young guinea fowl (keets): Nutrition study at village level. In : Compte rendu de l'atelier organisé sur le développement de la pintade en régions sèches africaines, Ouagadougou, Burkina Faso, 19-23 oct. 1992. Rome, Italie, $\mathrm{FAO}, 41 \mathrm{p}$.

10. DEFlY A., DOGBE A., ADMEFA K., 1999. Caractéristiques de I'aviculture traditionnelle. Etat actuel des connaissances au Togo et ailleurs. Lomé, Togo, Institut togolais de recherche agricole, $30 \mathrm{p}$.

11. DIABATE H., 1981. Elevage traditionnel de la pintade en Haute Volta. Mém., ISP, Université Ouagadougou, Burkina Faso, 109 p.

\section{Summary}

Sanfo R., Boly H., Sawadogo L., Brian O. Laying performances and egg characteristics of the guinea fowl (Numida meleagris) under improved breeding system in the central region of Burkina Faso

The laying performances and egg characteristics of 200 local guinea fowl (Numida meleagris) mated with 100 males were studied in Burkina Faso. The mean age at onset of lay was 5.7 months with a mean weight of $931.9 \pm 85.3 \mathrm{~g}$. The two laying cycles occurred in the dry seasons and lasted 4.8 months in the first dry season and 7.5 months in the second. Laying rates of 21.6 and $58.7 \%$, and average performances of 31.1 and 121.5 eggs per fowl, respectively, were recorded. Mean egg weights were $29.2 \pm 1.7 \mathrm{~g}$ in the first cycle vs $37.8 \pm 3.5 \mathrm{~g}$ in the second. Mean egg sizes were $47.1 \pm 1.9 \mathrm{~mm}$ in length and $36.5 \pm 1.3 \mathrm{~mm}$ in (large) diameter. The mean form index was higher in eggs of the second cycle than in those of the first $(0.79 \pm 0.05$ vs $0.76 \pm 0.02)$. The regression equation linking the egg weight $(\mathrm{Y})$ to the length $(\mathrm{L})$ and its large diameter $(\mathrm{Gd})$ was: $\mathrm{Y}(\mathrm{g})=-74.22+0.91 \mathrm{~L}(\mathrm{~mm})+1.83 \mathrm{Gd}(\mathrm{mm})\left(\mathrm{R}^{2}=0.96\right.$; standard error $=1.47$ ). Different shades of shells ( $\mathrm{red}=15.7 \%$; white $=19.7 \%$; tinted $=64.6 \%$ ) were identified for all eggs. The intrashell rate was $25.7 \%$ with $17.5 \%$ embryos and $8.2 \%$ chicks. Fertility and hatching rates of $82.7 \%$ and $74.3 \%$ were recorded, respectively.

Keywords: Poultry - Numida meleagris - Egg characters - Egg production - Laying performance - Burkina Faso.
12. GENDRON M., BLENTZ G., 1970. La qualité de I'œuf de consommation. Nouv. Avic. suppl. (125) : 1-28.

13. HIEN O.C., 2002. Effets de l'amélioration des conditions sanitaires sur le développement testiculaire, la LH et la ponte de la pintade locale au Burkina Faso. Thèse Doct., UFR-SVT, Université Ouagadougou, Burkina Faso, 126 p.

14. KUIT H.G., TRAORE A., WILSON R.T., 1986. Livestock production in Central Mali: Ownership, management and productivity of poultry in traditional sector. Trop. Anim. Health Prod., 18: 222-231.

15. OGUNTONA T., 1986. Two-year performances of guinea fowl layer fed proprietary layer rations. In: Proc. 9th Annu. Conf. Nigerian Society of Animal Production, University of Nigeria, Nsukka, 25-29 March 1984, p. $55-58$.

16. OLORI V., 1992. Composition and shell quality of the Nigerian indigenous guinea fowl In : Compte rendu de I'atelier organisé sur le développement de la pintade en régions sèches africaines, Ouagadougou, Burkina Faso, 19-23 oct. 1992. Rome, Italie, FAO, 41 p.

17. SANFO R., 2001. Productivité et variabilité individuelle du poulet local en conduites traditionnelle et améliorée au Centre du Burkina Faso. Mém. DEA, IDR, Bobo-Dioulasso, Burkina Faso, 40 p.

18. SANGARE M., 2005. Synthèse des résultats acquis en aviculture traditionnelle dans les systèmes de production animale d'Afrique de I'Ouest. Bobo-Dioulasso, Burkina Faso, Cirdes, 35 p.

19. SAUVEUR B., 1988. Reproduction des volailles et production d'œufs. Paris, France, Inra, 449 p.

20. SONAIYA E.B., 1992. La pintade : état des connaissances dans les zones arides à semi-humides d'Afrique de l'Ouest. In : Compte rendu de l'atelier organisé sur le développement de la pintade en régions sèches africaines, Ouagadougou, Burkina Faso, 19-23 oct. 1992. Rome, Italie, $\mathrm{FAO}, 41 \mathrm{p}$.

Accepté le 30.07.2012

\section{Resumen}

Sanfo R., Boly H., Sawadogo L., Brian O. Rendimiento de la postura y características de los huevos de la gallina pintada (Numida meleagris) bajo un sistema de manejo mejorado en la región central de Burkina Faso

En Burkina Faso fue realizado un estudio sobre el rendimiento de la postura y las características de los huevos de 200 gallinas pintadas (Numida meleagris) cubiertas por 100 machos. La edad promedio al inicio de la postura fue de 5,7 meses, con un peso promedio de 931,9 $\pm 85,3 \mathrm{~g}$. Los dos ciclos de postura ocurrieron durante las estaciones secas, con una duración de 4,8 meses para el primero y 7,5 meses para el segundo, con tasas de postura de 21,6 y $58,7 \%$, respectivamente, y un rendimiento promedio de 31,1 y 121,5 huevos por pintada. El peso promedio de los huevos fue 29,2 $\pm 1,7$ $\mathrm{g}$ en el primer ciclo y $37,8 \pm 3,5 \mathrm{~g}$ en el segundo. El tamaño promedio de los huevos fue 47,1 $\pm 1,9 \mathrm{~mm}$ de longitud $y$ $36,5 \pm 1,3 \mathrm{~mm}$ de diámetro grande. El índice promedio de la forma fue mayor en los huevos del segundo ciclo que en el primero $(0,79 \pm 0,05$ y $0,76 \pm 0,02)$. La ecuación de regresión con respecto al peso del huevo (Y), la longitud (L) y su diámetro grande (D) fue: $Y(\mathrm{~g})=-74,22+0,91 \mathrm{~L}(\mathrm{~mm})+1,83 \mathrm{D}$ $(\mathrm{mm})\left(\mathrm{R}^{2}=0,96\right.$, error estándar $\left.=1,47\right)$. Los tonos de colores de las cáscaras (pelirrojo $=15,7 \%$; blanco $=19,7 \%$; tintado $=64,6 \%$ ) se registraron para todos los huevos. La mortalidad dentro del huevo fue de $25,7 \%$ con $17,5 \%$ para los embriones y $8,2 \%$ para los pollitos. Se registraron tasas de fecundidad de $82,7 \%$ y tasas de eclosión de $74,3 \%$.

Palabras clave: Aves de corral - Numida meleagris - Características del huevo - Producción de huevos - Desempeño en la postura - Burkina Faso. 
